\title{
Role of surface roughness in superlubricity
}

\author{
U Tartaglino ${ }^{1,2}$, V N Samoilov ${ }^{1,3}$ and B N J Persson ${ }^{1}$ \\ ${ }^{1}$ IFF, FZ-Jülich, 52425 Jülich, Germany \\ ${ }^{2}$ Democritos National Simulation Center, Via Beirut 2, 34014 Trieste, Italy \\ ${ }^{3}$ Physics Faculty, Moscow State University, 117234 Moscow, Russia
}

Received 16 January 2006

Published 13 April 2006

Online at stacks.iop.org/JPhysCM/18/4143

\begin{abstract}
We study the sliding of elastic solids in adhesive contact with flat and rough interfaces. We consider the dependence of the sliding friction on the elastic modulus of the solids. For elastically hard solids with planar surfaces with incommensurate surface structures we observe extremely low friction (superlubricity), which very abruptly increases as the elastic modulus decreases. We show that even a relatively small surface roughness may completely kill the superlubricity state.
\end{abstract}

(Some figures in this article are in colour only in the electronic version)

\section{Introduction}

Friction between solid surfaces is a common phenomenon in nature and of extreme importance in biology and technology [1]. At the most fundamental level friction is (almost) always due to elastic instabilities at the sliding interface. At low sliding velocity an elastic instability first involves (slow) elastic loading, followed by a rapid rearrangement, where the speed of the rearrangement is much faster than, and unrelated to, the loading (or sliding) velocity. During the fast rearrangement the elastic energy gained during the loading phase is converted into irregular heat motion. The exact way the energy is 'dissipated' usually has a negligible influence on the sliding friction force, assuming that the dissipation occurs so fast that no memory of it remains during the next elastic loading event. There are many possible origins of elastic instability; e.g., it may involve individual molecules or, more likely, group of molecules or 'patches' at the interface which we have denoted by stress domains [2-5]. The most fundamental problem in sliding friction is to understand the physical origin and nature of the elastic instabilities.

Elastic instabilities occur only if the lateral corrugation of the interaction potential between the solid walls is high enough, or the elastic modulus of the solids small enough. Roughly speaking, elastic instabilities can only occur if a characteristic elastic energy is smaller than a characteristic binding (or rather barrier height) energy. To understand this, consider the simple model illustrated in figure 1. In (a) a particle or atom is moving in a corrugated (substrate) potential. Connected to the particle there is a spring (spring constant $k$ ) which is pulled with velocity $v$. If the spring is soft enough, or the potential barrier height $U$ is high enough, 

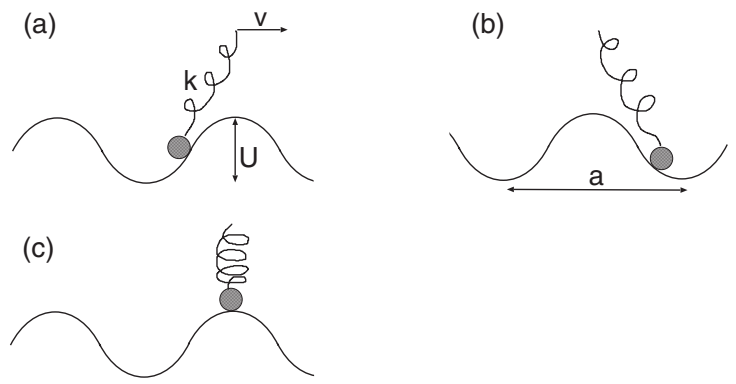

(d)

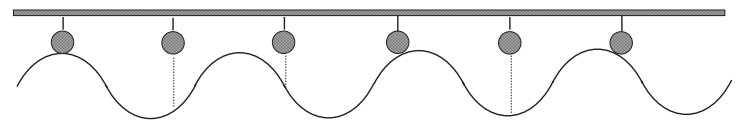

(e)

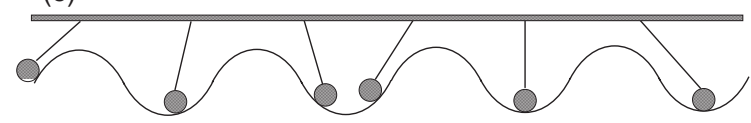

Figure 1. (a) A particle moving in a corrugated potential. The particle is connected to a spring which is pulled with velocity $v$. (b) Stick-slip motion with a soft spring, $k a^{2}<U$. (c) Continuous sliding with a stiff spring, $k a^{2}>U$. (d) Elastically stiff solid sliding on a rigid corrugated substrate. (e) Elastically soft solid sliding on a rigid corrugated substrate: here the atoms can rearrange to occupy binding positions.

i.e. $U>k a^{2}$, the particle will perform stick-slip motion (figures 1(a), (b)), involving slow elastic loading followed by rapid slip and dissipation of the (elastic) spring energy. In this case the (time averaged) force on the particle is independent of $v$. However, in the opposite case $U<k a^{2}$ (figure 1(c)), the particle will follow the drive with a velocity which is always comparable to $v$. In particular, when the drive is on top of the barrier so will the particle be (figure 1(c)). In this case no rapid motion will occur and the (time averaged) friction force acting on the particle is proportional to $v$.

In a more realistic situation one must consider the whole interface. In this case, depending on the elasticity and lateral barriers and the size of the contact area, elastic instabilities may or may not occur [6]. Assume first that an elastically very stiff solid slides on a rigid corrugated substrate (figure 1(d)). In this case the atoms at the bottom surface cannot adjust to the corrugated substrate potential, and (for an incommensurate system) as some atoms move downhill other atoms move uphill in such a way that the total energy is constant. Thus, no elastic instabilities will occur during sliding, resulting in a very low sliding friction; this state has been termed superlubric [7]. However, when the block is elastically soft (figure 1(e)), the atoms can rearrange themselves so that at any moment in time almost all the atoms occupy positions close to the minima of the substrate potential. During sliding, rapid jumps will occur from time to time where a particle changes potential well. In this case the friction is high and (at zero temperature) remains finite as the sliding velocity $v \rightarrow 0$.

It is well known that elastically hard solids tend to exhibit smaller sliding friction than (elastically) soft materials [8]. One extreme example is diamond, which under normal circumstances exhibits very low kinetic friction coefficient, of the order of 0.01 , when diamond is sliding on diamond. This can be explained by the near absence of elastic instabilities because of the elastic hardness of the material. However, if clean diamond is sliding on clean diamond 


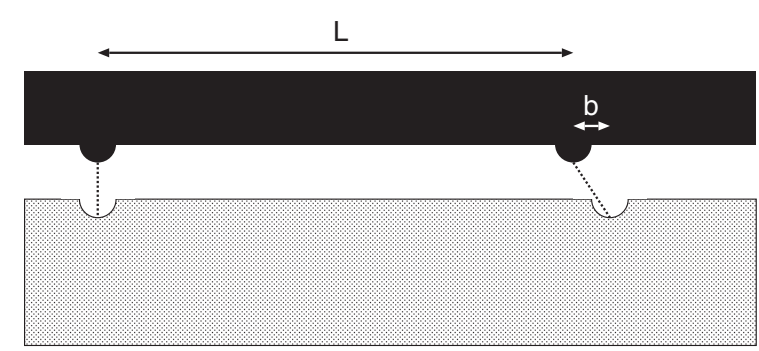

Figure 2. An elastic bar with two binding sites (bump). The corresponding binding cavities of the substrate do not match exactly the positions of the binding sites, causing a competition between the energy cost to stretch the elastic bar and the binding energy.

in ultrahigh vacuum, a huge friction (friction coefficient of the order of $\mu \approx 10$ ) is observed [9]. The reason is that the clean surfaces have dangling bonds (which are passivated by hydrogen and oxygen in the normal atmosphere) so that the interaction between the two diamond surfaces is very strong and elastic instabilities (and wear processes) can occur, resulting in a very large friction.

It is important to note that even if solids are too stiff for elastic instabilities to occur on short length scale the ratio between the effective elasticity and the amplitude of the lateral corrugation of the binding potential may decrease when the system is studied at a longer length scale, which may make elastic instabilities possible on a longer length scale [10,11]. To illustrate this, in figure 2 we show a one-dimensional (1D) case, where an elastic bar (cross section area $A$ ) with two binding sites (bumps) is in contact with a substrate with two binding sites (cavities). When the binding sites on the elastic bar overlap with the binding sites (cavities) on the (rigid) substrate, the binding energy $U$ is gained. In order to gain this binding energy the segment of the elastic bar between the bumps (length $L$ ) must elongate by the distance $b$. Thus the strain in the segment is $b / L$ and the elastic energy stored in the elongated segment is $U_{\mathrm{el}}=V E(b / L)^{2} / 2$, where the volume $V=L A$. Thus, $U_{\mathrm{el}}=A E b^{2} /(2 L)$, which decreases as the length of the segment $L$ increases. It follows that only when $L>A E b^{2} /(2 U)$ will the bound state have a lower energy than the non-bound state. Thus, only on a large enough length scale will the solid be elastically soft enough for elastic instabilities to occur. In most practical cases one is not interested in a 1D situation but rather in semi-infinite solids, which are intermediate between the $2 \mathrm{D}$ and $3 \mathrm{D}$ case. For surfaces with randomly distributed binding centres this situation is much more complex than for the 1D case because the effective elasticity changes as quickly with the lateral length scale as does the effective amplitude of the lateral corrugation of the binding potential (which from random walk arguments [1] scales as $L)[10,11]$. A detailed analysis of this situation indicates, however, that if no elastic instability can occur at short length scale it is very unlikely that elastic instabilities will occur on any length scale of practical importance, except perhaps in the context of earthquakes $[10,11]$. If instead of randomly distributed binding sites one assumes incommensurate surfaces, one would expect even weaker pinning effects, and it can be argued that in this case the ratio between the effective elasticity and the amplitude of the lateral corrugation of the binding potential increases as $\sim L$ so that if no elastic instabilities occur at short length scale they cannot occur at any length scale [12]. Below we will present numerical results where elastic instabilities do occur also for (nearly) incommensurate structures, but in these cases one of the solids is elastically very soft so that instabilities can occur on a short length scale.

The discussion above has focused on clean surfaces and zero temperature. Temperature is unlikely to have any drastic influence on superlubricity. However, it may have a strong influence 
on sliding dynamics when elastic instabilities occur. As soon as $T>0 \mathrm{~K}$, thermal noise is able to activate jumps over the barrier, i.e. to provoke premature jumps before the (zero-temperature) instability point is reached. It has been shown experimentally [5, 13] and theoretically [3, 14] that this has a crucial influence on friction dynamics at low sliding velocity. Similarly, weakly bound adsorbed atoms and molecules have a large influence on the sliding dynamics, and may strongly increase the friction force [15] as the mobile adsorbates can adjust themselves in the corrugated potential between the block and the substrate, giving rise to strong pinning effects. In this paper we will not address the role of adsorbates or non-zero temperature, but we will focus on the simplest case of clean surfaces at zero temperature.

Recently, superlubricity has been observed during sliding of graphite on graphite: in the experiment described in [16] a tungsten tip with a graphite flake attached to it is slid on an atomically flat graphite surface. When the flake is in registry with the substrate stick-slip motion and large friction are observed. When the flake is rotated out of registry, the forces felt by the different atoms start to cancel each other out, causing the friction force to nearly vanish, and the contact to become superlubric.

Graphite and many other layered materials are excellent dry lubricants. The most likely reason for this is that the solid walls of the sliding objects get coated by graphite flakes or layers with different orientation so a large fraction of the graphite-graphite contacts will be in the superlubric state. This will lead to a strong reduction in the average friction. However, the coated solid walls are unlikely to be perfectly flat and it is important to address how surface roughness may influence the superlubric state. In this paper we will show that even a relatively small surface roughness may kill the superlubric state.

Lubrication by graphite flakes may even occur for diamond-like carbon (DLC) coatings, which may exhibit very low friction. Indeed, Liu et al [17] have observed that a graphitized tribolayer is formed on top of diamond-like carbon coatings. Thus, the excellent lubrication properties of DLC films might also be caused by superlubric graphite contacts. We also note that DLC films are very hard and this will reduce the chance for elastic instabilities to occur ${ }^{4}$.

In this paper we present atomistic molecular dynamics calculations of the sliding dynamics for contacting elastic solids with (nearly) incommensurate surface lattice structures. We consider both flat and rough surfaces. We consider the dependence of the sliding friction on the elastic modulus of the solids. For elastically hard solids with flat surfaces and incommensurate surface structures we observe extremely low friction (superlubricity), which very abruptly increases as the elastic modulus is diminished. We show that even a small surface roughness may completely kill the superlubric state. In order to study large systems we use a recently developed multiscale approach [19] to contact mechanics where the number of dynamical variables scales like $\sim N^{2}$ rather than as $\sim N^{3}$, where $N \times N$ is the number of atoms in the nominal contact area.

\section{Multiscale molecular dynamics}

Let us discuss the minimum block size necessary in a computer simulation for an accurate description of the contact mechanics between two semi-infinite elastic solids with nominally

\footnotetext{
4 The properties of diamond-like carbon (DLC) films depend strongly on the preparation method and operation conditions. Thus, only DLC films produced from discharge plasmas containing much hydrogen will exhibit a low friction $(\mu \sim 0.001-0.003)$. This is believed to result from the passivation of carbon dangling bonds by the hydrogen atoms. Without hydrogen, in an inert atmosphere the friction is huge (of the order of unity) because of a high concentration of very reactive carbon dangling bonds. In the normal atmosphere, most dangling bonds are passivated and the friction lower but still much higher than for diamond or for DLC films produced from plasmas containing much hydrogen. See [18].
} 
(a)
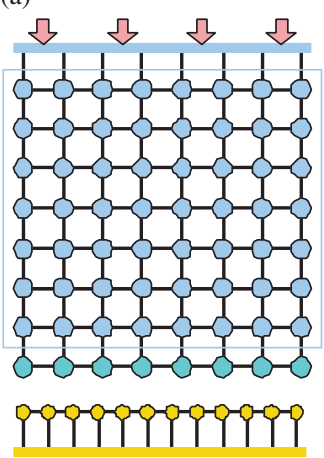

(b)

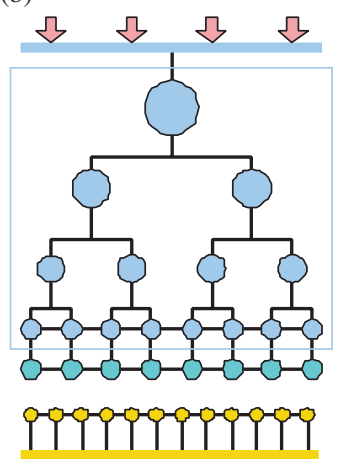

Figure 3. Schematic structure of the model. (a) The fully atomistic model. (b) The multiscale smartblock model, where the solid in (a) is coarse grained by replacing groups of atoms with bigger 'atoms'.

flat surfaces. Assume that the surface roughness power spectrum has a roll-off wavevector $q=q_{0}$ corresponding to the roll-off wavelength $\lambda_{0}=2 \pi / q_{0}$. In this case the minimum block must extend $L_{x} \approx \lambda_{0}$ and $L_{y} \approx \lambda_{0}$ along the $x$ and $y$ directions. Furthermore, the block must extend at least a distance $L_{z} \approx \lambda_{0}$ in the direction perpendicular to the nominal contact area, since the surface roughness with wavelength $\lambda_{0}$ affects the elastic block up to such a distance. Thus, the minimum block is a cube with the side $L=\lambda_{0}$.

As an example, if $\lambda_{0}$ corresponds to 1000 atomic spacings, one must at least consider a block with $1000 \times 1000$ atoms within the $x y$ contact plane, i.e., one would need to study the elastic deformations in a cubic block with at least $10^{9}$ atoms. However, it is possible to drastically reduce the number of dynamical variables without loss of accuracy if one notes that an interfacial roughness with wavelength $\lambda$ will give rise to a deformation field in the block which extends a distance $\lambda$ into the solid, and which does not have any significant variation over distances much smaller than $\lambda$. Thus when we study the deformation a distance $z$ into the block we do not need to describe the solid on the atomistic level, but we can coarse-grain the solid by replacing groups of atoms with bigger 'atoms' as indicated schematically in figure 3 . If there are $N \times N$ atoms in the nominal contact area one need $n \approx \ln N$ 'atomic' layers in the $z$-direction. Moreover the number of atoms in each layer decreases in a geometric progression every time the coarse graining procedure is applied, so that the total number of particles is of the order of $N^{2}$ instead of $N^{3}$. This results in a huge reduction in the computational time for large systems. This multiscale approach may be implemented in various ways, and in [19] we outline the procedure we have used in this paper, which we refer to as the smartblock.

The model presented above should accurately describe the deformations in the solids as long as the deformations vary slowly enough with time. However, the phonons with short wavelength cannot propagate in the coarse grained region because the model does not implement enough degrees of freedom for them. The short wavelength phonons get scattered back when they reach the coarse grained region, and this cannot be avoided within a standard molecular dynamics approach. However, this is not a serious limitation for the present work: the static friction and the onset of sliding are still unaffected; moreover, the superlubric sliding at low speed does not dissipate energy into phonons. On the other hand, if one had to study the spectrum of dissipated phonons, more advanced techniques to adsorb the energy of the phonons without back scattering have to be considered [20,21]. The only dissipation mechanism that we employed in our simulations is a Langevin thermostat at $T=0 \mathrm{~K}$ (i.e. a viscous friction term) acting only on the atoms of the block far from the contact region. 
Figure 3 illustrates a case where the block is in the form of a cube with atomically flat surfaces. It is possible to obtain curved surfaces of nearly arbitrary shape by 'gluing' the upper surface of the block to a hard curved surface profile. This was described in detail in [22]. The elastic modulus and the shear modulus of the solid can be fixed at any value by proper choice of the elongation and bending spring constants for the springs connecting the atoms (see [22] and [19]).

We note that with respect to contact mechanics, when the slopes of the surfaces are small, i.e. when the surfaces are almost horizontal, one of the two surfaces can be considered flat, while the profile of the other surface has to be replaced by the difference of the two original profiles [23]. Thus, if the substrate has the profile $z=h_{1}(\mathbf{x})$ and the block has the profile $z=h_{2}(\mathbf{x})$, then we can replace the actual system with a fictive one where the block has an atomically smooth surface while the substrate profile $h(\mathbf{x})=h_{2}(\mathbf{x})-h_{1}(\mathbf{x})$. Furthermore, if the original solids have the elastic modulus $E_{1}$ and $E_{2}$, and the Poisson ratio $v_{1}$ and $v_{2}$, then the substrate in the fictive system can be treated as rigid and the block as elastic with the elastic modulus $E$ and Poisson ratio $v$ chosen so that $\left(1-v^{2}\right) / E=\left(1-v_{1}^{2}\right) / E_{1}+\left(1-v_{2}^{2}\right) / E_{2}$.

The results presented below have been obtained for an elastic flat block sliding on a rigid substrate. We considered both flat and rough substrates. The atoms in the bottom layer of the block form a simple square lattice with lattice constant $a$. The lateral dimensions $L_{x}=N_{x} a$ and $L_{y}=N_{y} a$. For the block, $N_{x}=N_{y}=48$. Periodic boundary conditions are applied in the $x y$ plane. The lateral size of the block is equal to that of the substrate, but for the latter we use a different lattice constant $b \approx a / \phi$, where $\phi=(1+\sqrt{5}) / 2$ is the golden mean, in order to avoid the formation of commensurate structures at the interface. For the substrate, $N_{x}=N_{y}=78$. The mass of a block atom is $197 \mathrm{amu}$ and the lattice spacing of the block is $a=2.6 \AA$, to get the same atomic mass and density of gold. The lattice spacing of the substrate is $b=1.6 \AA$. We consider solid blocks with different Young's moduli from $E=0.2 \mathrm{GPa}$ up to $1000 \mathrm{GPa}$. The Poisson ratio used for the block is $v=0.3$.

The atoms at the interface between the block and the substrate interact with the potential

$$
U(r)=4 \epsilon\left[\left(\frac{r_{0}}{r}\right)^{12}-\alpha\left(\frac{r_{0}}{r}\right)^{6}\right],
$$

where $r$ is the distance between a pair of atoms. When $\alpha=1$, equation (1) is the standard Lennard-Jones potential. The parameter $\epsilon$ is the binding energy between two atoms at the separation $r=2^{1 / 6} r_{0}$. When we study contact mechanics and friction without adhesion we put $\alpha=0$. In the calculations presented below we have used $r_{0}=3.28 \AA$ and $\epsilon=40 \mathrm{meV}$, which (when $\alpha=1$ ) gives an interfacial binding energy (per unit area) [24] $\Delta \gamma \approx 4 \epsilon / a^{2} \approx 23.7 \mathrm{meV}^{-2}$.

As an illustration, in figure 4 we show the contact between a flat elastic block (top) and a randomly rough rigid substrate (bottom). Only the interfacial block and substrate atoms are shown. The substrate is a self-affine fractal with the root-mean-square roughness $3 \AA$ (see section 3). Note the elastic deformation of the block, and that non-contact regions occur in the 'deep' valleys of the substrate. Actually, the real contact area is smaller than the nominal contact area.

\section{Self-affine fractal surfaces}

Consider a solid with a nominally flat surface. Let $x, y, z$ be a coordinate system with the $x, y$ plane parallel to the surface plane. Assume that $z=h(\mathbf{x})$ describes the surface height profile, where $\mathbf{x}=(x, y)$ is the position vector within the surface plane. The most important property characterizing a randomly rough surface is the surface roughness power spectrum $C(\mathbf{q})$ defined 


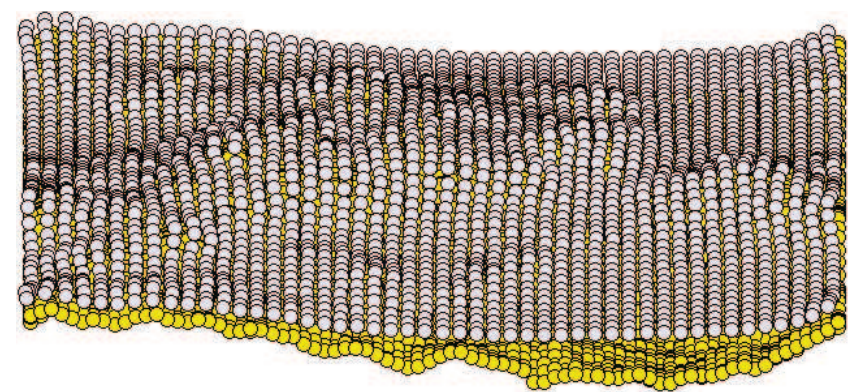

Figure 4. The contact between an elastic block with a flat surface and a rough rigid substrate. Only the interfacial layers of atoms are shown. The elastic modulus of the block is $E=100 \mathrm{GPa}$. The substrate is a self-affine fractal with the root-mean-square roughness $3 \AA$, fractal dimension $D_{\mathrm{f}}=2.2$ and roll-off wavevector $q_{0}=3 q_{L}$, where $q_{L}=2 \pi / L_{x}$. The substrate and block interfacial atomic layers consisted of $78 \times 78$ and $48 \times 48$ atoms, respectively. The applied pressure $p=10 \mathrm{GPa}$. Note the elastic deformation of the block, and that the real contact area is smaller than the nominal contact area.

by $[22,25]$

$$
C(\mathbf{q})=\frac{1}{(2 \pi)^{2}} \int \mathrm{d}^{2} x\langle h(\mathbf{x}) h(\mathbf{0})\rangle \mathrm{e}^{\mathrm{iq} \cdot \mathbf{x}} .
$$

Here $\langle\cdots\rangle$ stands for the ensemble average and we have assumed that $h(\mathbf{x})$ is measured from the average surface plane so that $\langle h\rangle=0$. In what follows we will assume that the statistical properties of the surface are isotropic, in which case $C(q)$ will only depend on the magnitude $q=|\mathbf{q}|$ of the wavevector $\mathbf{q}$.

Many surfaces tend to be nearly self-affine fractal. A self-affine fractal surface has the property that if part of the surface is magnified, with a magnification which in general is appropriately different in the perpendicular direction to the surface as compared to the lateral directions, then the surface 'looks the same', i.e., the statistical properties of the surface are invariant under this scale transformation [25]. For a self-affine surface the power spectrum has the power-law behaviour

$$
C(q) \sim q^{-2(H+1)},
$$

where the Hurst exponent $H$ is related to the fractal dimension $D_{\mathrm{f}}$ of the surface via $H=$ $3-D_{\mathrm{f}}$. Of course, for real surfaces this relation only holds in some finite wavevector region $q_{0}<q<q_{1}$, and in a typical case $C(q)$ has the form shown in figure 5. Note that in many cases there is a roll-off wavevector $q_{0}$ below which $C(q)$ is approximately constant.

In our calculations we have used self-affine fractal surfaces generated as outlined in [25]. Thus, the surface height is written as

$$
h(\mathbf{x})=\sum_{\mathbf{q}} B(\mathbf{q}) \mathrm{e}^{\mathrm{i}[\mathbf{q} \cdot \mathbf{x}+\phi(\mathbf{q})]},
$$

where, since $h(\mathbf{x})$ is real, $B(-\mathbf{q})=B(\mathbf{q})$ and $\phi(-\mathbf{q})=-\phi(\mathbf{q})$. If $\phi(\mathbf{q})$ are independent random variables, uniformly distributed in the interval $[0,2 \pi[$, then one can easily show that higher order correlation functions involving $h(\mathbf{x})$ can be decomposed into a product of pair correlations, which implies that the height probability distribution $P_{h}=\langle\delta(h-h(\mathbf{x}))\rangle$ is Gaussian [25]. However, such surfaces can have arbitrary surface roughness power spectrum. To prove this, substitute (3) into (2) and use that

$$
\left\langle\mathrm{e}^{\mathrm{i} \phi\left(\mathbf{q}^{\prime}\right)} \mathrm{e}^{\mathrm{i} \phi\left(\mathbf{q}^{\prime \prime}\right)}\right\rangle=\delta_{\mathbf{q}^{\prime}},-\mathbf{q}^{\prime \prime}
$$




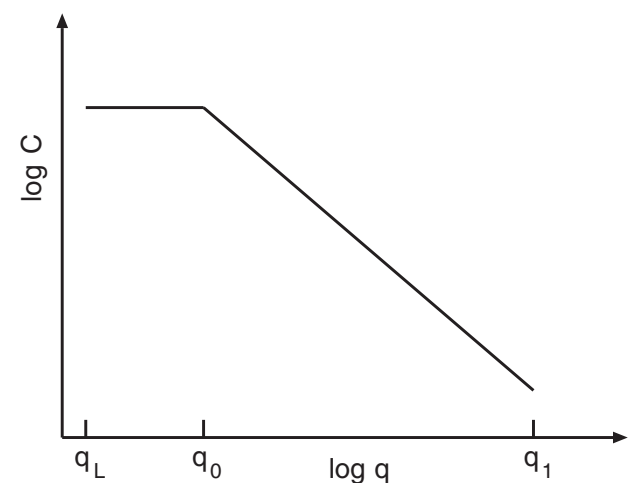

Figure 5. Surface roughness power spectrum of a surface which is a self-affine fractal for $q_{0}<q<q_{1}$. The long-distance roll-off wavevector $q_{0}$ and the short distance cut-off wavevector $q_{1}$ depend on the system under consideration. The slope of the $\log C-\log q$ relation for $q>q_{0}$ determines the fractal exponent of the surface. The lateral size $L$ of the surface (or of the studied surface region) determines the smallest possible wavevector $q_{L}=2 \pi / L$.

gives

$C(\mathbf{q})=\frac{1}{(2 \pi)^{2}} \int \mathrm{d}^{2} x \sum_{\mathbf{q}^{\prime}}\left|B\left(\mathbf{q}^{\prime}\right)\right|^{2} \mathrm{e}^{\mathrm{i}\left(\mathbf{q}-\mathbf{q}^{\prime}\right) \cdot \mathbf{x}}=\sum_{\mathbf{q}^{\prime}}\left|B\left(\mathbf{q}^{\prime}\right)\right|^{2} \delta\left(\mathbf{q}-\mathbf{q}^{\prime}\right)$.

Replacing

$$
\sum_{\mathbf{q}} \rightarrow \frac{A_{0}}{(2 \pi)^{2}} \int \mathrm{d}^{2} q
$$

where $A_{0}$ is the nominal surface area, gives

$$
C(\mathbf{q})=\frac{A_{0}}{(2 \pi)^{2}}|B(\mathbf{q})|^{2} .
$$

Thus, if we choose

$$
B(\mathbf{q})=(2 \pi / L)[C(\mathbf{q})]^{1 / 2},
$$

where $L=A_{0}^{1 / 2}$, then the surface roughness profile (3) has the surface roughness power spectrum $C(\mathbf{q})$. If we assume that the statistical properties of the rough surface are isotropic, then $C(\mathbf{q})=C(q)$ is a function of the magnitude $q=|\mathbf{q}|$, but not of the direction of $\mathbf{q}$. The randomly rough substrate surfaces used in our numerical calculations were generated using (3) and (4) and assuming that the surface roughness power spectra have the form shown in figure 5, with the fractal dimension $D_{\mathrm{f}}=2.2$ and the roll-off wavevector $q_{0}=3 q_{L}$, where $q_{L}=2 \pi / L_{x}$. We have chosen $q_{0}=3 q_{L}$ rather than $q_{0}=q_{L}$ since the former value gives some self-averaging and less noisy numerical results. We also used $q_{1}=2 \pi / b=78 q_{0}$. The topography of the substrate with the root-mean-square roughness amplitude $3 \AA$ used in our numerical calculations is shown in figure 6.

\section{Numerical results}

In this section we present the results of molecular dynamics calculations of sliding of elastic blocks on rigid substrates. In all cases, unless otherwise stated, the upper surface of the block moves with the velocity $v=0.1 \mathrm{~m} \mathrm{~s}^{-1}$, and the (nominal) squeezing pressure $p$ is one-tenth of the elastic modulus $E$ of the block, i.e. $p=0.1 E$. The reason for choosing $p$ proportional to 


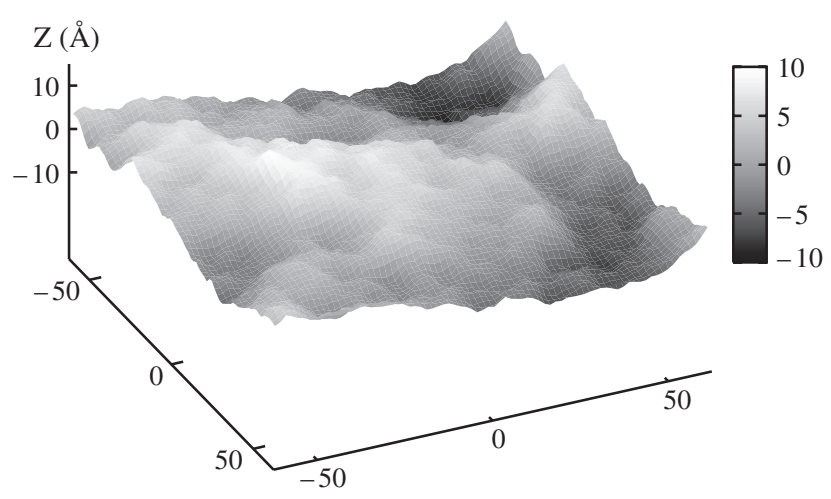

Figure 6. Fractal surface of the substrate with the large cut-off wavevector $q_{1}=2 \pi / b=78 q_{0}$. For a square $124.8 \AA \times 124.8 \AA$ surface area. The fractal dimension $D_{\mathrm{f}}=2.2$ and the root-mean-square roughness amplitude is $3 \AA$.

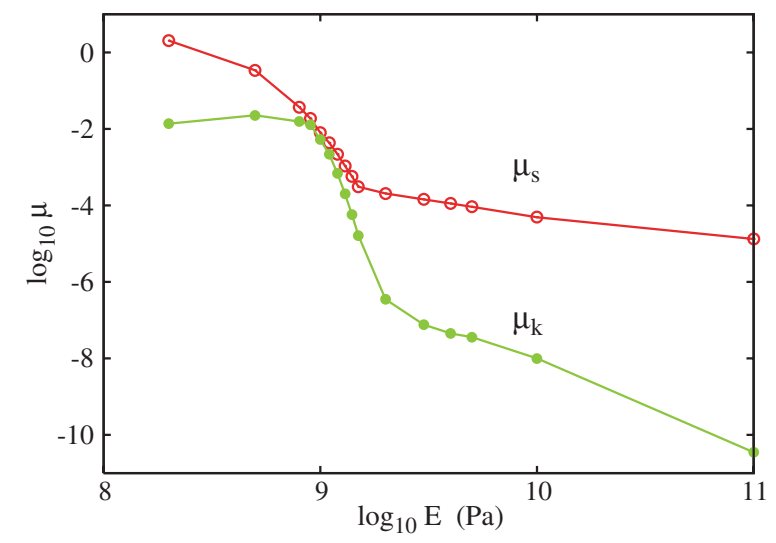

Figure 7. The static (curve 1) and kinetic (curve 2) friction coefficients as a function of the elastic modulus $E$ of the block, for the flat substrate. In the calculation we have assumed the squeezing pressure $\sigma_{0}=0.1 E$ and the sliding velocity $v=0.1 \mathrm{~m} \mathrm{~s}^{-1}$.

$E$ is twofold. First, we consider solids with elastic modulus which varies over several orders of magnitude, and it is not possible to use a constant $p$ as this would result in unphysical large variations in the elastic deformation of the block. Second, if two elastic solids are squeezed together with a given load, then as long as the area of real contact is small compared to the nominal contact area the pressure in the contact areas will be proportional to the elastic modulus of the solids [22]. Initially, when the block is pulled laterally it deforms loading elastic energy and the shear force between the surfaces increases gradually. The shear force reaches a maximum $F_{\mathrm{S}}$ at the onset of sliding. We used such a maximum shear force to calculate the static friction coefficient: $\mu_{\mathrm{s}}=F_{\mathrm{s}} / F_{\mathrm{N}}, F_{\mathrm{N}}=p A$ being the normal force. During the sliding the shear force oscillates in time. We defined the kinetic friction coefficient as the ratio between the time averaged shear force and the normal load, i.e. $\mu_{k}=\overline{F_{k}} / F_{\mathrm{N}}$.

Let us first assume that both the block and the substrate have atomically smooth surfaces. Figure 7 shows the static and the kinetic friction coefficients as a function of the elastic modulus $E$ of the block. Note the relatively abrupt decrease in the friction when the elastic modulus changes from $E_{1} \approx 0.7 \mathrm{GPa}$ to $E_{2} \approx 2 \mathrm{GPa}$. For $E>E_{2}$ practically no instabilities occur and 

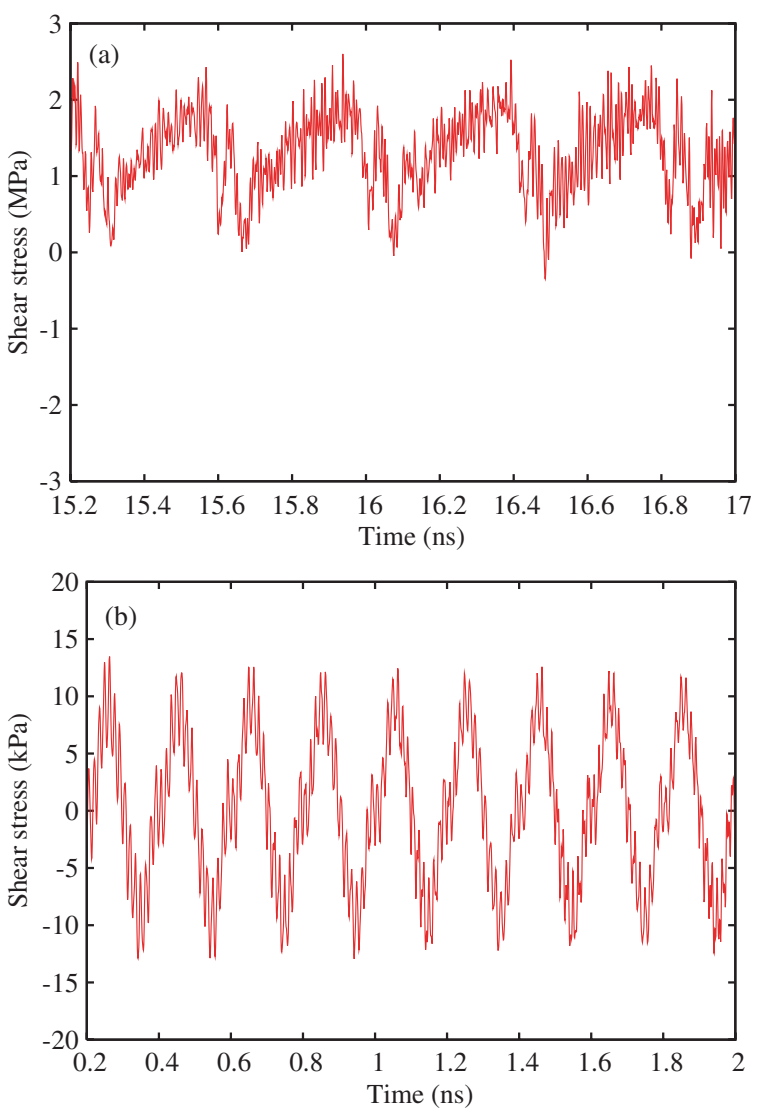

Figure 8. (a) The shear stress as a function of time for the flat substrate. The elastic modulus of the block is $E=0.8 \mathrm{GPa}$. (b) The same as above but for the elastic modulus of the block $E=2 \mathrm{GPa}$.

the friction is extremely small, while for $E<E_{1}$ relatively strong elastic instabilities occur at the sliding interface, and the friction is high. For $E=0.2 \mathrm{GPa}$ the static friction $\mu_{\mathrm{s}}>2$. This calculation illustrates that the transition from high friction to superlubricity can be very abrupt; in the present case an increase in the elastic modulus by only a factor of $\sim 3$ (from 0.7 to $2.1 \mathrm{GPa}$ ) decreases the kinetic friction by a factor of $\sim 10^{5}$.

In figure 8 we show the time variation of the shear stress as a function of time when the elastic modulus of the block equals (a) $E=0.8 \mathrm{GPa}$ and (b) $E=2 \mathrm{GPa}$. The elastic modulus of the stiffer solid is above the superlubricity threshold, and no (or negligible) elastic instabilities occur; the stress is a periodic function of time, with the period corresponding to the displacement $0.2 \AA$. For the softer solid strong elastic instabilities occur during sliding, the shear stress is less regular (and the arrangement of the interfacial block atoms more disordered) than for the stiffer solid, and the (average) period is longer than $0.2 \AA$.

The regular pattern with period $0.2 \AA$ in figure 8 (b) can be understood as follows. For our system, in the sliding direction there are eight block atoms for every 13 substrate atoms. Assume first that the block (and the substrate) are perfectly stiff. In this case, the position of the eight block atoms will take eight uniformly spaced positions within the substrate unit cell (lattice constant $b$ ); see figure 9. Thus, a shift of the block with the distance $b / 8$ will take the system to a (geometrically) equivalent configuration. Hence, since $b=1.6 \AA$ we expect the 

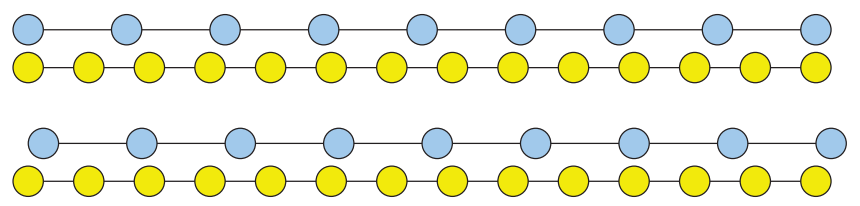

Figure 9. Commensurability ratio $8 / 13$ between one-dimensional chains. The upper image shows the period of eight block atoms (light blue), i.e. 13 substrate atoms (yellow). The lower image is obtained by shifting the block for $1 / 13$ of its lattice spacing. Block atoms occupy the same positions relatively to the substrate's hollows.

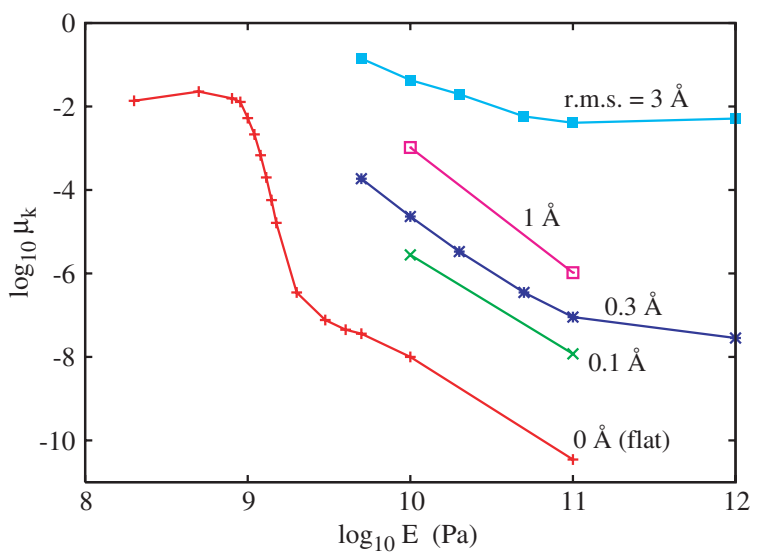

Figure 10. The kinetic friction coefficients for an elastic block sliding on rough substrates, as a function of the logarithm of elastic modulus $E$ of the block. Curves 1-5 (from top to bottom) correspond to the root-mean-square roughness amplitudes of the fractal substrate 3,1,0.3,0.1 $\AA$ and 0 (flat substrate).

periodicity of the shear stress to be $b / 8=0.2 \AA$. When the block has a finite elasticity but above the superlubricity threshold, the atoms will relax somewhat in the substrate potential, but the configuration of the system will still repeat itself with the same period $b / 8$.

Let us now consider the influence of surface roughness on the sliding dynamics. In figure 10 we show the kinetic friction coefficients for an elastic block sliding on a rough substrate, as a function of the logarithm of elastic modulus $E$ of the block. The curves from top to bottom correspond to the substrate root-mean-square roughness amplitudes $3,1,0.3$, $0.1 \AA$ and 0 (flat substrate). For the substrate with the largest roughness, no superlubricity state can be observed for any elastic modulus up to $E=10^{12} \mathrm{~Pa}$.

In figure 11 we show the kinetic friction coefficient as a function of the root-mean-square roughness amplitude of the substrate. The elastic modulus of the block $E=100 \mathrm{GPa}$. Note the strong decrease in the friction when the root-mean-square roughness amplitude decreases below $0.3 \AA$, which corresponds to a peak-to-peak roughness of roughly one atomic lattice spacing.

Figure 12(a) shows the average (or nominal) shear stress as a function of time for the rough substrate with the root-mean-square roughness $3 \AA$, and for the elastic modulus of the block $E=100,50$ and $20 \mathrm{GPa}$. Note that in addition to major slip events several small slip events occur in all cases. These events correspond to local slip at some asperity contact regions before the major slip involving the whole contact area. In all cases, the time dependence of the shear stress remains periodic with the period 2.6 $\AA$, which corresponds to the lattice spacing of the 


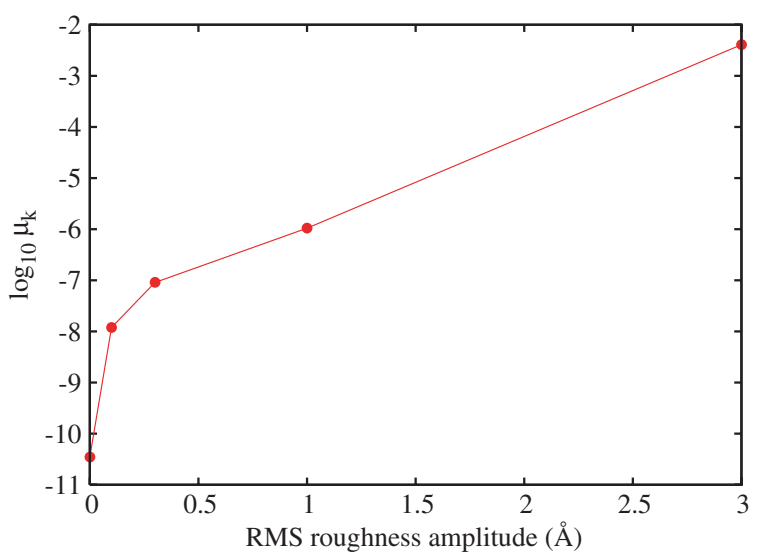

Figure 11. The kinetic friction coefficient as a function of the root-mean-square roughness amplitude of the substrate. The elastic modulus of the block $E=100 \mathrm{GPa}$.
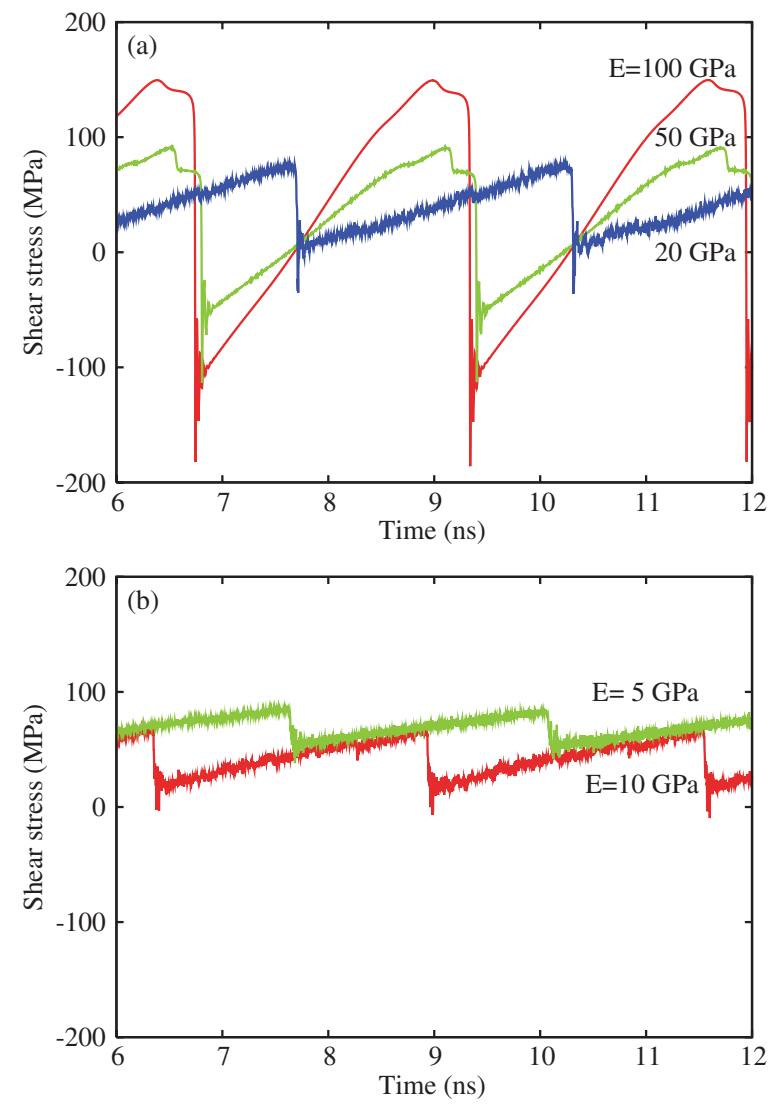

Figure 12. (a) The shear stress as a function of time for the rough substrate with root-mean-square roughness amplitude $3 \AA$. The elastic modulus of the block is $E=100,50$ and $20 \mathrm{GPa}$. (b) The same as above but for the elastic modulus of the block $E=10$ and $5 \mathrm{GPa}$. 


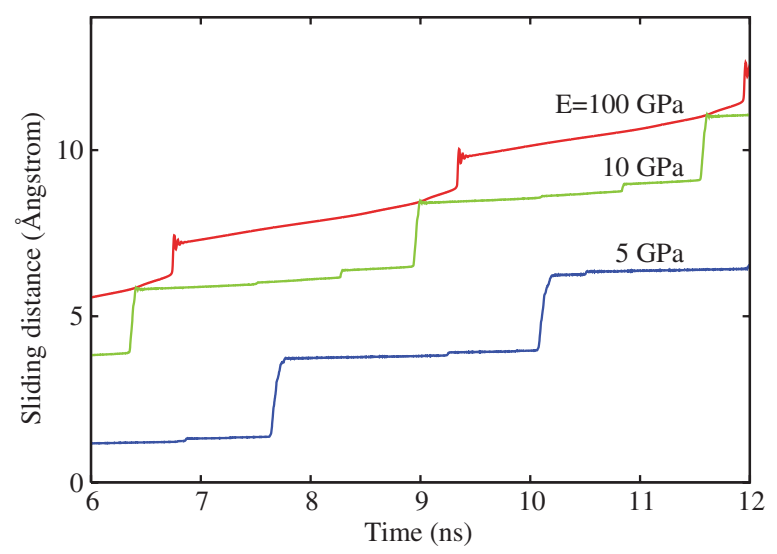

Figure 13. The average displacement of the interfacial atoms of the block as a function of time. The root-mean-square roughness amplitude for the substrate is $3 \AA$. The elastic modulus of the block is $E=100,10$ and $5 \mathrm{GPa}$.

block. Note also that for the elastically softer block ( $E=20 \mathrm{GPa})$ the stress noise increases after each major slip event; this is caused by the elastic waves (heat motion) excited during the (major) rapid slip events and not completely adsorbed by the thermostat.

Figure 12(b) shows the same as in (a) but now for the elastic modulus of the block $E=10$ and $5 \mathrm{GPa}$. In this case the decrease of the elastic modulus of the block results in the increase of both the static and kinetic friction.

Figure 13 shows the average displacement of the interfacial atoms of the block (in the sliding direction) as a function of time. The root-mean-square roughness amplitude for the substrate is $3 \AA$. The elastic modulus of the block is $E=100,10$ and $5 \mathrm{GPa}$. Note that the slip distance for the major slip events increases as the elastic modulus of the block decreases, and that for the elastically hardest solid $\left(E=10^{11} \mathrm{~Pa}\right)$ about a half of the forward displacement occurs between the major slip events.

Figure 14 shows the average position of the interface block atoms in the $z$-direction (perpendicular to the sliding direction) as a function of time. Results are shown for the rough substrate with the root-mean-square roughness amplitude $3 \AA$. The elastic modulus of the block is $E=100,50,20,10$ and $5 \mathrm{GPa}$. When the elastic modulus decreases, because of the adhesive interaction the block interfacial atoms come (on average) closer to the rigid substrate, embracing the substrate asperities. This increases the real area of contact between the surfaces and results in a higher friction.

Figure 15(a) shows the shear stress as a function of time for the rough substrate (rootmean-square amplitude $3 \AA$ ) and for the stiff block ( $E=100 \mathrm{GPa})$. The solid curve is with the adhesion included, while the dashed curve is without the attractive part in the Lennard-Jones potential, i.e. with $\alpha=0$ in equation (1). Note that without adhesion the major slip is not so pronounced as for the case with adhesion. Still the time dependence of the shear stress remains periodic with the same period $2.6 \AA$, corresponding to the lattice spacing of the block. Without adhesion, the shear stress curve is nearly symmetric around the zero-stress axis, and the kinetic friction coefficient (determined by the average shear stress divided by the squeezing pressure) is about 150 times smaller than when the adhesive interaction is included.

In figure 15(b) we show the average displacement of the interface block atoms (in the sliding direction) as a function of time for the same systems as in (a). For the case without 


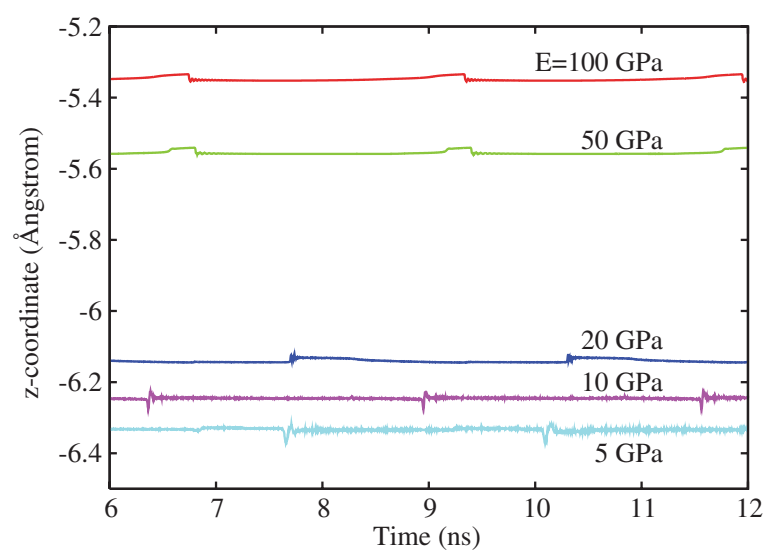

Figure 14. The average position of the interface block atoms in the $z$-direction (perpendicular to the sliding direction), as a function of time during sliding. The root-mean-square roughness amplitude of the substrate is $3 \AA$. The curves from top to bottom correspond to the elastic modulus of the block $E=100,50,20,10$ and $5 \mathrm{GPa}$.

adhesion the major slip is not as abrupt as when adhesion is included. At every moment there is some lateral motion of the block interfacial atoms.

Figure 16(a) shows the shear stress as a function of time for the rough substrate (rootmean-square amplitude $3 \AA$ ) and for the elastic block with the elastic modulus $E=10 \mathrm{GPa}$. The solid curve is with adhesion included, while the dashed curve is without the attractive part in the Lennard-Jones potential, i.e. with $\alpha=0$ in equation (1). Figure 16(b) shows the average displacement of the interface block atoms (in the sliding direction) as a function of time for the same systems as in (a). For the case without adhesion the major slip is not as abrupt as for the case with adhesion, and the sliding motion is nearly steady. In both cases, the time dependence of shear stress remains periodic with the period $2.6 \AA$ determined by the lattice spacing of the block. For the case with adhesion two small slips and a major slip can be observed in each period, and the kinetic friction is high. For the case without adhesion no elastic instability occurs, and the kinetic friction is very small.

The roughness-induced increase of friction can be understood by considering that the real contact involves only small regions, as shown in figure 4 . The compensation of the lateral forces that guarantees superlubricity between incommensurate surfaces (see figure 1) does not happen at the boundaries of the contact regions, nor can it happen on very small contacts with high curvature. Thus, the friction force should increase with increasing length of the boundaries between the contact and non-contact regions. This is completely different from what happens between commensurate walls or between walls with very strong interactions, e.g. metals with cold-welded microjunctions, where the real area of contacts determines the friction force.

The surfaces used in our simulations are self-affine fractals up to the atomic scale, but in general the cut-off wavevector $q_{1}$ can be smaller, so that the typical size of the contact areas can be larger and the friction can be lower. In other words, the root-mean-square surface roughness alone is not enough to determine the amount of friction: a surface profile with wider mountains and valleys has to provide less friction than a surface profile dominated by short wavelength corrugation. In the same way, a surface with higher fractal dimension will have more roughness at the smallest wavelength, providing higher friction for the same root-mean-square roughness and cut-off wavevector $q_{1}$. In particular for high fractal dimensions the friction must depend dramatically on the cut-off wavevector. 

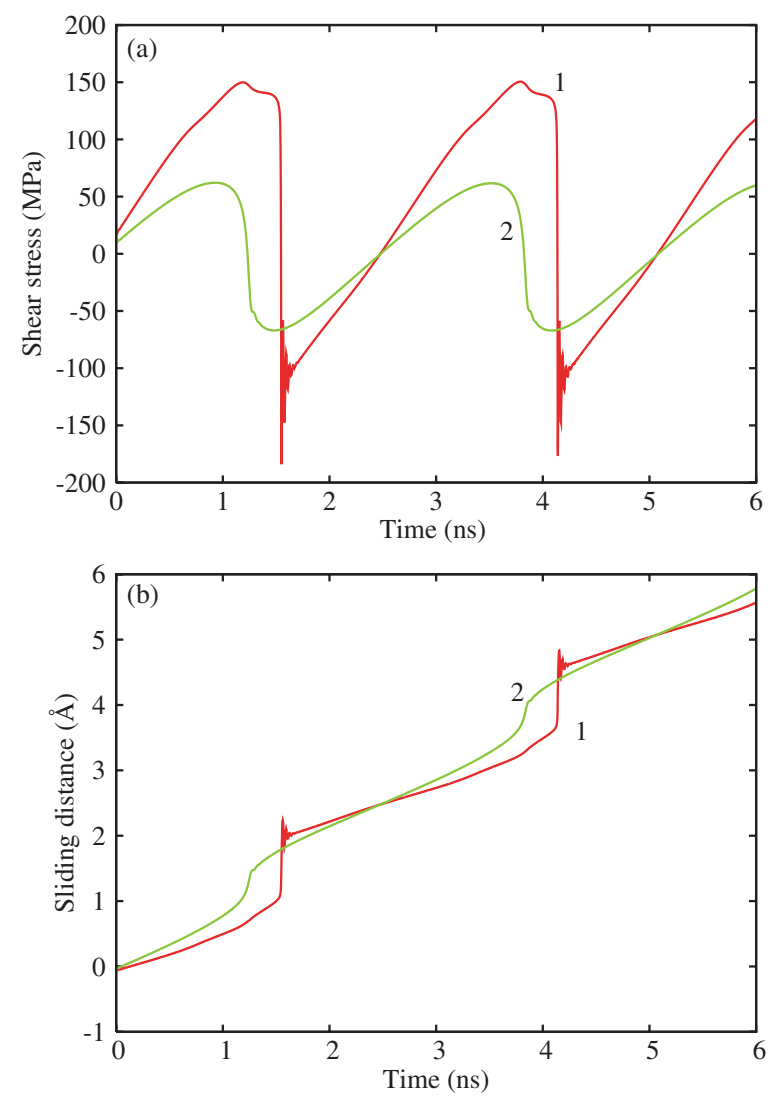

Figure 15. (a) The shear stress as a function of time calculated for the rough substrate (root-meansquare amplitude $3 \AA)$ and for the stiff block $(E=100 \mathrm{GPa})$. Curve 1 is calculated including adhesion, while curve 2 is obtained without the attractive part in the Lennard-Jones potential, i.e. with $\alpha=0$ in equation (1). (b) The average displacement of the interface block atoms (in the sliding direction) as a function of time for the same systems as in (a).

\section{Pressure dependence of the frictional stress}

During sliding, the atoms at the sliding interface will experience energetic barriers derived from both the adhesive interaction between the atoms on the two opposing surfaces, and from the applied load. Thus, we may define an adhesion pressure $p_{\text {ad }}$, and as long as $p_{\text {ad }} \gg p$, where $p$ is the pressure in the contact area derived from the external load, the frictional shear stress will be nearly independent of the applied load. Let us illustrate this with the system studied in section 4. Let us first consider the limiting case where the elastic modulus of the block is extremely small. In this case, in the initial pinned state (before sliding) all the block atoms will occupy hollow sites on the substrate, as indicated by atom $\mathbf{A}$ in figure 17. During sliding along the $x$-direction, the atom $\mathbf{A}$ will move over the bridge position $\mathbf{B}$ and then 'fall down' into the hollow position $\mathbf{C}$ (we assume overdamped motion). The minimum energy for this process is given by the barrier height $\delta \epsilon$ (the energy difference between the sites $\mathbf{B}$ and $\mathbf{A}$ ) plus the work $p a^{2} \delta h$ against the external load, where $a$ is the block lattice constant and $\delta h$ the change in the height between sites $\mathbf{B}$ and $\mathbf{A}$ (which depends on $p$ ). Thus the frictional shear stress $\sigma_{\mathrm{f}}$ is 

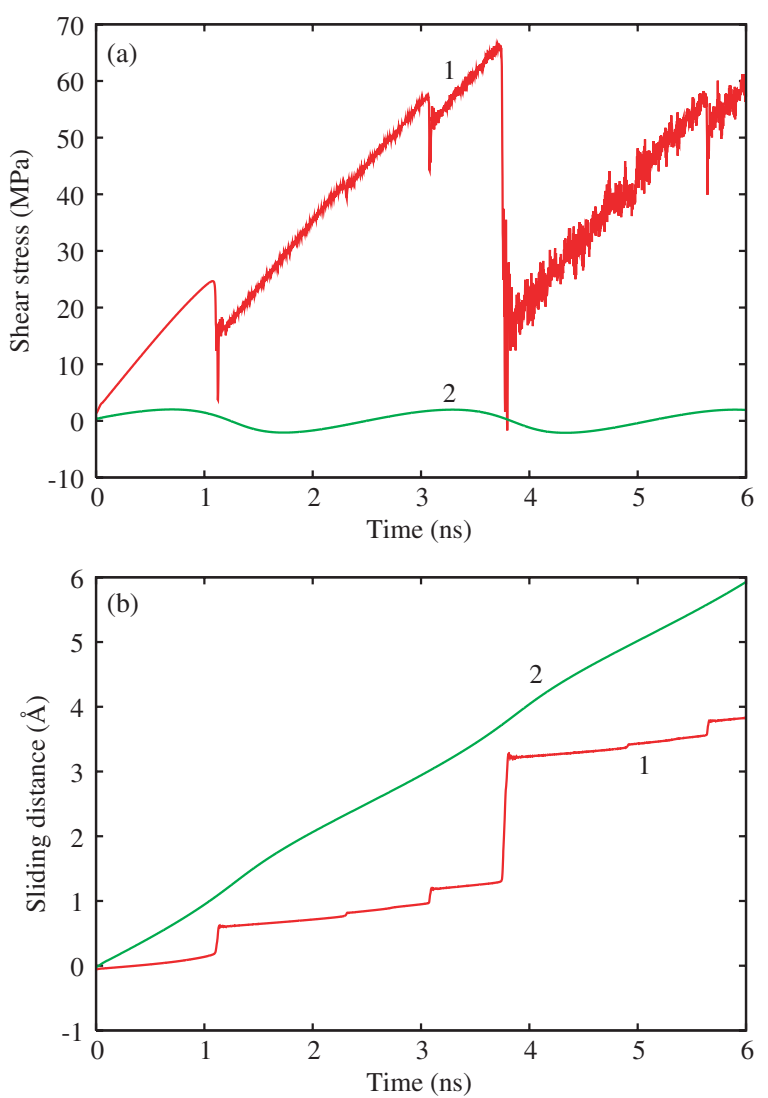

Figure 16. (a) The shear stress as a function of time for the rough substrate (root-mean-square amplitude $3 \AA$ ) and for the block elastic modulus $E=10 \mathrm{GPa}$. Curve 1 is calculated including adhesion, while curve 2 is obtained without the attractive part in the Lennard-Jones potential, i.e. with $\alpha=0$ in equation (1). (b) The average displacement of the interface block atoms (in the sliding direction) as a function of time for the same systems as in (a).

determined by $\sigma_{\mathrm{f}} a^{2} b=\delta \epsilon+p a^{2} \delta h$, or

$$
\sigma_{\mathrm{f}}=\delta \epsilon /\left(b a^{2}\right)+p \delta h / b=\left(p_{\mathrm{ad}}+p\right) \delta h / b,
$$

where we have defined the adhesion pressure $p_{\text {ad }}=\delta \epsilon /\left(a^{2} \delta h\right)$.

In our case $\delta \epsilon \approx 3 \mathrm{meV}$ and $\delta h \approx 0.008 \AA$ giving $p_{\text {ad }} \approx 10^{10} \mathrm{~Pa}$. Thus, in the present case, only when the local pressure in the contact regions becomes of the order of $\sim 10 \mathrm{GPa}$, or more, will it start to influence the shear stress. This result is in accordance with our simulation results. Thus, for smooth surfaces, the shear stress acting on the block with the elastic modulus $E=0.5 \mathrm{GPa}$, squeezed against the substrate with the pressure $p=50$ and $150 \mathrm{MPa}$, is identical $(\approx 1 \mathrm{MPa})$ within the accuracy of the simulations.

For inert materials such as rubber the adhesive pressure may be of similar magnitude as obtained above. Since the contact pressure for rubber in most cases is below $10 \mathrm{MPa}$, one may expect that the shear stress in the areas of real contact will be independent of the load. Recently, a strong dependence of the (apparent) shear stress on the squeezing pressure was observed for smooth Plexiglas balls sliding on very smooth silicon wafers covered by silane layers [26]. However, as one of us has argued elsewhere [27], this does not reflect a fundamental dependence of the shear stress on the squeezing pressure, but has another origin. 


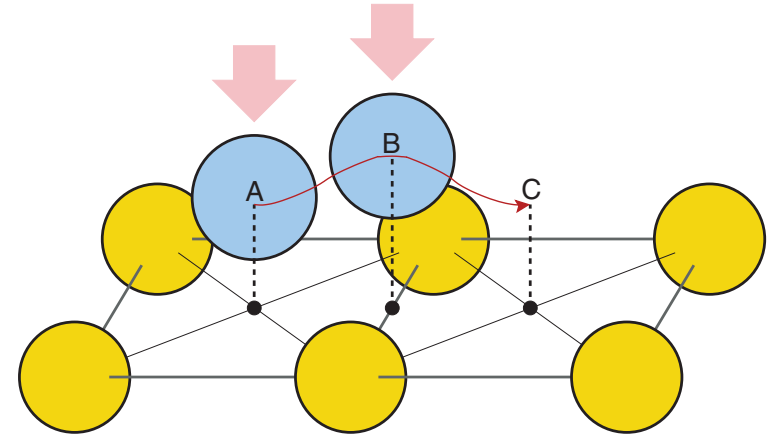

Figure 17. A block atom moving (or jumping) from the hollow site $\mathbf{A}$ over the bridge site $\mathbf{B}$ to the hollow site $\mathbf{C}$. The maximum energy position along the trajectory is at site $\mathbf{B}$.

\section{Summary and conclusion}

We have studied the sliding of elastic solids in adhesive contact with flat and rough interfaces. We considered the dependence of the sliding friction on the elastic modulus of the solids. For elastically hard solids with planar surfaces with incommensurate surface structures we observe extremely low friction (superlubricity), which very abruptly increases as the elastic modulus decreases. Thus, at the superlubricity threshold, an increase in the elastic modulus by a factor of $\sim 3$ resulted in the decrease in the frictional shear stress by a factor $\sim 10^{5}$. We have shown that even a relatively small surface roughness may completely kill the superlubricity. For flat surfaces the shear stress is independent of the perpendicular (squeezing) pressure as long as the pressure $p$ is below the adhesive pressure $p_{\text {ad }}$, which typically is of the order of several GPa.

\section{Acknowledgments}

Part of the present work was carried out in the frame of the ESF programme "Nanotribology (NATRIBO)'. Two of the authors (UT and VNS) acknowledge support from IFF, FZ-Jülich, and hospitality and help of the staff during their research visits. The authors also thank V V Samoilov for technical assistance with preparing some figures for the paper. This work was partly sponsored by MIUR FIRB RBAU017S8 R004, MIUR FIRB RBAU01LX5H, MIUR COFIN 2003 and PRIN-COFIN 2004.

\section{References}

[1] Persson B N J 2000 Sliding Friction: Physical Principles and Applications 2nd edn (Heidelberg: Springer)

[2] Persson B N J, Albohr O, Mancosu F, Peveri V, Samoilov V N and Sivebaek I M 2003 Wear 254835

[3] Persson B N J 1995 Phys. Rev. B 5113568

[4] Caroli C and Nozieres P 1998 Eur. Phys. J. B 4233

[5] Baumberger T and Caroli C 2005 Preprint cond-mat/0506657 v1

[6] Aubry J 1983 J. Physique 44147

[7] Shinjo K and Hirano M 1993 Surf. Sci. 283473

[8] Riedo E and Brune H 2003 Appl. Phys. Lett. 831986

[9] van der Oetelaar R J A and Flipse C F J 1997 Surf. Sci. 384 L828

[10] Persson B N J and Tosatti E 1999 Solid State Commun. 109739

[11] Caroli C and Nozieres P 1996 Physics of Sliding Friction ed B N J Persson and E Tosatti (Dordrecht: Kluwer)

[12] Müser M H 2004 Europhys. Lett. 6697 
[13] Gnecco E, Bennewitz R, Gyalog T, Loppacher Ch, Bammerlin M, Meyer E and Güntherodt H-J 2000 Phys. Rev. Lett. 841172

Riedo E, Gnecco E, Bennewitz R, Meyer E and Brune H 2003 Phys. Rev. Lett. 91084502

[14] Sang Y, Dube M and Grant M 2001 Phys. Rev. Lett. 87174301

[15] He G, Müser M H and Robbins M O 1999 Science 2841650

[16] Dienwiebel M, Verhoeven G S, Pradeep N, Frenken J W M, Heimberg J A and Zandbergen H W 2004 Phys. Rev. Lett. 92126101

[17] Liu Y, Erdemir A and Meletis E I 1996 Surf. Coat. Technol. 86/87 564

[18] See Erdemir A 2001 Surf. Coat. Technol. 146/147 292

[19] Yang C, Tartaglino U and Persson B N J 2006 Eur. Phys. J. E 1947

[20] Cai W, de Koning M, Bulatov V V and Yip S 2000 Phys. Rev. Lett. 853213

[21] E W and Huang Z 2001 Phys. Rev. Lett. 87135501

[22] Persson B N J 2001 J. Chem. Phys. 1153840

[23] Johnson K L 1985 Contact Mechanics (Cambridge: Cambridge University Press)

[24] Israelachvili J 1992 Intermolecular and Surface Forces (London: Academic)

[25] Persson B N J, Albohr O, Tartaglino U, Volokitin A I and Tosatti E 2005 J. Phys.: Condens. Matter 17 R1

[26] Bureau L, Baumberger T and Caroli C 2006 Eur. Phys. J. E 19163 (Preprint cond-mat/0510232 v1)

[27] Persson B N J 2006 Surf. Sci. Rep. at press 